\title{
A coupled volume of fluid-fictitious domain method to study droplet-particle interactions at different impact conditions
}

\author{
Mahdi Saeedipour*1, Achuth Nair Balachandran Nair ${ }^{1}$, Stefan Pirker ${ }^{1}$ \\ ${ }^{1}$ Department of Particulate Flow Modelling, Johannes Kepler University, Linz, Austria \\ ${ }^{*}$ Corresponding author email: mahdi.saeedipour@jku.at
}

\begin{abstract}
In this study, we present a coupled volume of fluid-fictitious domain method for the simulation of droplet-particle binary interactions. In this approach, the liquid-gas flow is modelled by the VOF method, and the particle presence is introduced to the Eulerian grids by a volume fraction scalar field. The hydrodynamic forces acting on the particle are computed over the particlecovered grids, and particle moves according to Newton's second law. A penalization term is added to the momentum equation at the particle-covered grids via a continuous force field. The VOF algorithm is then modified to prevent the penetration of liquid into the particle region. First, a 3D benchmark problem is tested for validation. Then, a 3D configuration is considered for the droplet-particle interaction where a water droplet and a solid particle constitute a headson binary collision in the surrounding air. The collision behaviour at different impact velocities and droplet-to-particle diameter ratios are pictured, and the outcome regimes are characterized based on impact Weber numbers. The present approach is developed within the opensource $\mathrm{C}++$ libraries of OpenFOAM and LIGGGHTS ${ }^{\circledR}$ codes and can be further employed for the fully-resolved description of the interfacial physics in droplet-laden flows in the presence of moving particles.
\end{abstract}

\section{Keywords}

VOF, immersed boundary method, penalization method, droplet-particle interactions, multiphase flow

\section{Introduction}

Atomization, fluidization, and emulsification are examples of dispersed multiphase flows that are present in different chemical, pharmaceuticals, and metallurgical industries. There are also situations where the dispersed phase contains both fluid and solid elements, e.g. liquid droplets and solid particles. In such multiphase systems, the binary interaction of droplets and particles is important for various applications including, but not limited to, spray drying [1], particle capture in spray towers [2], spray coating in spouted beds [3], and particle encapsulation [4]. From a mathematical modelling viewpoint and depending on the target physical scales, most of these applications can be described by Eulerian one-fluid and twofluid formulations of the two-phase flows as well as Eulerian-Lagrangian methods. However, a small-scale picture of the liquid-gas flows in the presence of solid particles, as happens in droplet-particle collisions, requires extra treatment to resolve the hydrodynamic forces on the particle and the fluid-solid interactions. This justifies the use of immersed boundary-based methods [5] where fixed Eulerian grids are used to solve the fluid fields, and the particle presence imposes a rigid body constraint to the fluid flow. There are two approaches to account for this constraint: (I) the direct forcing method that imposes a no-slip boundary condition at the rigid boundaries [6], and (ii) the continuous forcing method (also known as fictitious domain) that introduces a penalization term for the rigid domain in the momentum equation before discretization [7]. While the immersed boundary method is originally designed for single-phase flows in the presence of rigid bodies, its combination with multiphase flow simulation methods, mainly interfacial flows, has also gained attention in the past years. 
Particularly, efforts have been made in the context of liquid-gas flows with particles [8-12]. Apart from the method, numerical investigations on the binary interaction of particles and droplets are rather scarce, unlike the droplet-droplet collisions. Malgarinos et al. [13] simulated the droplet-particle collisions at low and moderate Weber numbers (We). They studied the wetting area over particles and characterized the outcome regimes in a map for different We and droplet-to-particle diameter ratios. Bordbar et al. [14], Vilela, and de Souza [15] studied the outcome of droplet-particle impact employing Level set and VOF simulations, respectively. The former remains limited to the low Weber number collisions, and the latter only focuses on the physics of lamella formation after the impact. Milacic et al. [16] performed a detailed simulation of droplet spreading on the spherical particle. Recently, Yoon and Shin [17] reported a DNS study on the droplet collision with stationary particles unveiling new postimpact regimes. Most of these methods are computationally expensive for large-scale systems. Besides, neither of them uses the immersed boundary method (except [16] which is limited to very low Weber number collision), and the stationary particle is considered as a wall boundary in the domain. Thus, if multiscaling [18] is intended, their applicability to small-scale fully-resolved simulations of more dynamic systems might be limited. Especially, when the outcome of droplet-particle collision is subject to more interactions with other particles and droplets. The main objective of the present study is to provide a numerical platform where such a droplet-particle collision could be simulated at higher We numbers (more than 100) with a reasonable computational cost. The combination of VOF with the DEM-based fictitious domain in the present study enables such simulations and has a direct implication to multiscale methods and magnification lens concept.

\section{Numerical method description}

The fluid dynamics of the droplet-particle interactions can be described by a two-phase liquidgas flow (droplet in surrounding gas) in the presence of a moving solid region (particle). Therefore, the mathematical formulation of the fluid flow in such a three-phase system follows a coupling concept between these two physical environments. The governing equations of two-phase incompressible flow in the context of one-fluid formulation consist of the continuity and Navier-Stokes equations together with the advection equation for VOF function as follows:

$$
\begin{gathered}
\frac{\partial \rho}{\partial t}+\nabla \cdot(\rho U)=0 \\
\frac{\partial(\rho U)}{\partial t}+\nabla \cdot(\rho U \otimes U)=-\nabla p+\nabla \cdot(2 \mu D)+\rho g+F_{\sigma}+F_{p} \\
\frac{\partial \alpha}{\partial t}+\nabla \cdot(\alpha U)=0
\end{gathered}
$$

In this formulation, $U$ is the mixture velocity vector shared with both fluid phases, and the scalar $\alpha$ is the volume fraction which is one at the liquid region and zero in the gas, and determines the physical properties of the fluid. For more details, we refer to [19]. Besides the fluid flow, Newton's second law governs the motion of the solid particles, which reads

$$
\begin{gathered}
m_{p} \frac{d U_{p}}{d t}=m_{p} g+\sum F_{p}^{f} \\
I_{p} \frac{d \omega_{p}}{d t}=r \times \sum F_{p}^{f}
\end{gathered}
$$

Where $F_{p}^{f}$ represents the fluid hydrodynamic forces acting on the particles in these force and tourqe equations. More details on these equation can be found in [20]. For coupling the particle 
dynamics with the volume of fluid method, we follow the resolved CFD-DEM approach [20], which is a combination of immersed boundary concept with the discrete element method. In this approach, the particle is treated as an immersed body whose diameter is much larger than the computational grid size. Since the three phases share the same computational domain, another scalar field is defined as the particle volume fraction $(\Phi)$, which is one at the particle region and zero elsewhere. This enables to detect the particle-covered cells and to provide a fully-resolved picture of the fluid-particle interactions by including a rigid body constraint in the fluid domain, for which there are two approaches as explained in the introduction. The direct forcing approach imposes a no-slip boundary condition at the particle boundary and makes the corrections during pressure-velocity coupling to keep the velocity field divergence-free [2021]. The continuous forcing method (fictitious domain) introduces a source term in the momentum equation before discretization. The former is employed in the original resolved CFD-DEM solver of CFDEMcoupling [20] for the typical single-phase flow coupled with resolvable particles. However, for the two-phase flows coupled with solid particles, we employ the latter because it reduces the complexity of velocity correction during the pressure-velocity coupling in the presence of liquid volume fraction. Therefore, the source term $F_{p}$ in equation (2) is defined as a low permeable porous region [22] that reads

$$
F_{p}=\rho \Phi \frac{\left(U_{p}-U\right)}{\kappa \Delta t}
$$

where $k$ is the permeability of the solid region that is chosen very small $(k=1 e-7)$ [22] to avoid the penetration of the fluid into the particle region. The dependency of the forcing term to the $\backslash \mathrm{PHI}$ ensures that the rigid body constraint is only imposed at particle region (i.e. $\Phi=1$ ). A VOF solver using PISO algorithm for pressure-velocity coupling technique then solves this system of equations numerically.

\section{Method implementation and solver development}

The described coupled volume of fluid-fictitious domain method is implemented as an open source solver using the $\mathrm{C}_{+}+$libraries of OpenFOAM (www.openfoam.org) and LIGGGHTS (www.cfdem.com). Particularly, we coupled interFoam, the VOF solver of OpenFOAM, with the DEM solver as schematically shown in Figure 1. The CFD part of the solver is responsible for the interface capturing of the liquid-gas flow in the presence of rigid body constraint.

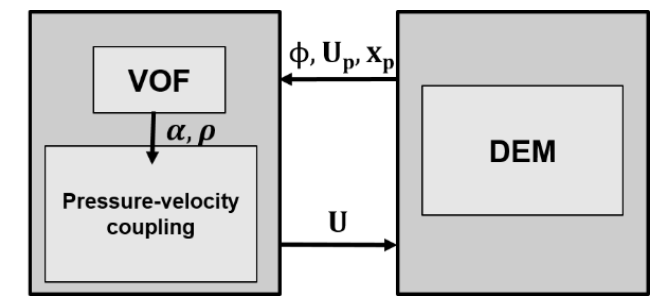

Figure 1. Schematic of the developed VOF-DEM solver.

The hydrodynamic body and surface forces acting on the particle are computed via a coupling library, while the DEM part of the solver updates the particle position and velocity based on Newton's second law. Thus, the particle position and velocity vectors are updated. This information is then passed to the CFD part to determine (I) the new $\Phi$ field and (II) compute the forcing term in equation (6), and (III) to solve the equations for $\alpha, U$ and pressure fields in the fluid regions. For (I), the finite volume cells occupied by the solid particle are identified by a 
smooth particle representation algorithm [21]. For (II) an explicit source term is computed in the momentum equation, and finally for (III) the computed flux in each finite volume cell is multiplied by $(1-\Phi)$ to ensure that no mass and momentum transport occurs in the particlecovered fictitious domain. To guarantee the mass conservation, further modification is required at the end of VOF loop in each time step as follows:

$$
\rho=(1-\Phi)\left[\alpha \rho_{1}+(1-\alpha) \rho_{2}\right]+\Phi \rho_{p}
$$

This ensures that the density of the particle is also included in the total mass conservation system. It has to be noted that in the present study the wettability of the particle is not considered. It is assumed that a zero-gradient condition (similar to $90^{\circ}$ contact angle) for the liquid volume fraction must be fulfilled upon contact of liquid-gas interface with the particle.

\section{Validation study}

In order to validate the present numerical method a water entry problem is selected which was subject to an experimental study by Aristoff et al., [23] and also used in [24] for the validation purpose. In this benchmark problem, a solid particle is initially set at the top of a water pool as schematically described in Figure 2 . A three-dimensional geometry $\left(\mathrm{L}_{x}=80 \mathrm{~mm}, \mathrm{~L}_{y}=120\right.$ $\mathrm{mm}$ and $\mathrm{L}_{z}=80 \mathrm{~mm}$ ) is created as the computational domain, with no-slip boundary condition at all the surrounding walls except the top patch which is an open to atmosphere boundary. The domain was discretized uniformly with two different grid resolutions of $\Delta \mathbf{x}_{1}=1 \mathrm{~mm}$ and $\Delta \mathrm{x}_{2}=0.66 \mathrm{~mm}$. They are corresponding to 26 and 40 cells per particle diameter, respectively. The water and air properties at room temperature are used for the two-phase flow $\left(\rho_{w}=1000\right.$ $\mathrm{kg} / \mathrm{m}^{3}, \rho_{\mathrm{a}}=1 \mathrm{~kg} / \mathrm{m}^{3}, \mu_{\mathrm{w}}=1 \mathrm{e}-3$ Pa.s, $\mu_{\mathrm{a}}=1.5 \mathrm{e}-5$ Pa.s, and $\left.\sigma=0.072 \mathrm{~N} / \mathrm{m}\right)$.
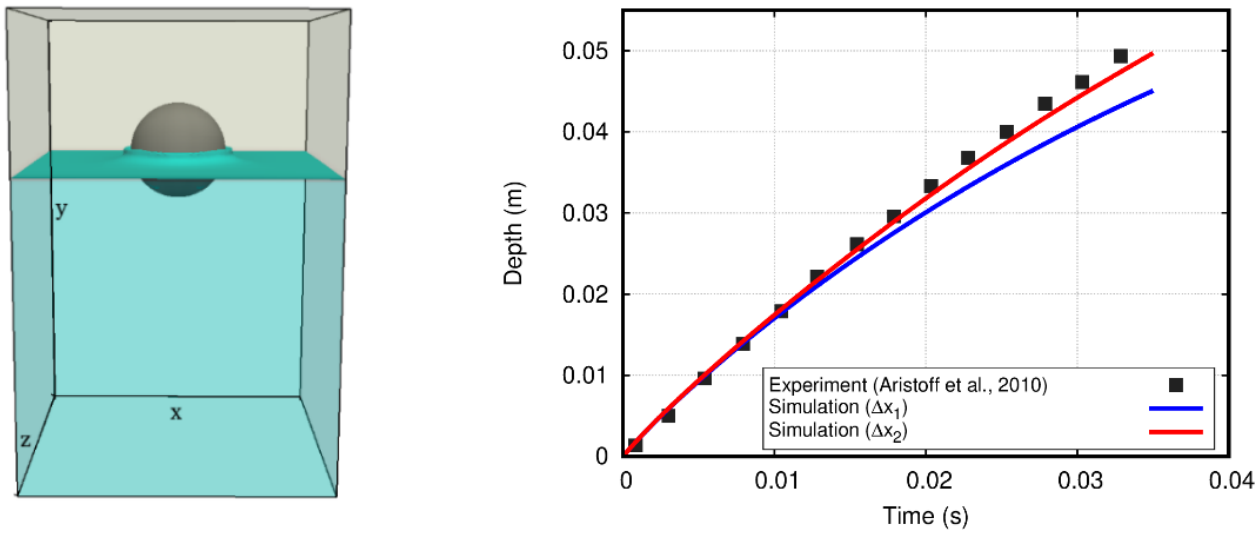

Figure 2. The simulation setup for the validation case (left), and the result of particle depth into the water from experiment and simulations.

The particle diameter and density are $25.5 \mathrm{~mm}$ and $860 \mathrm{~kg} / \mathrm{m} 3$, respectively. An initial velocity of $2.17 \mathrm{~m} / \mathrm{s}$ is set for the particle. As the validation measure, Figure 2 compares the particle depth over time obtained by experiment and simulation. It is evident that the simulation results converge to the experimental data for $\Delta \mathrm{x}_{2}$. The slight discrepancy at latest stage might be related to the velocity measurement error during the experiment as also discussed in [24].

\section{Simulation setup for droplet-particle interaction}

To study the binary collision of a water droplet and solid particle, a three-dimensional geometry $\left(L_{x}=100 \mathrm{~mm}, L_{y}=50 \mathrm{~mm}\right.$, and $\left.L_{z}=50 \mathrm{~mm}\right)$ is created which is uniformly discretized with structural grids of $\Delta x=0.66 \mathrm{~mm}$ (similar to the grid size for the converged case in the validation 
study). As schematically described in Figure 3, the droplet and particle are initially located on the same axis with the opposite initial velocity of $U_{0}$, resulting in a relative velocity of $2 U_{0}$. The material properties of the water and surrounding air are chosen similar to the validation case. We assume $\rho_{d}=\rho_{p}$ to avoid density contrast effects during the impact. To account for different impact conditions, the Weber number $\left(W e=\frac{\rho U_{r e l}^{2} D_{d}}{\sigma}\right)$ and the droplet-to-particle diameter ratio $\left(R=D_{d} / D_{p}\right)$ are varied. Table 1 presents the simulation cases with all the input parameters. For $R=1$, we assume equal diameters of $D_{d}=D_{p}=20 \mathrm{~mm}$. For $R>1$, the size of the particle is reduced, while for $R<1$ we reduced the droplet size. Although the We number might slightly decrease in the latter case, it helps to perform all the simulation cases on the same computational domain. Depending on the impact velocity, some of the cases were simulated for a longer time until the collision and post-collision physics are sufficiently established. Each simulation took between 2-3 hours using 32 cores of our in-house computational cluster. For a better comparison between the cases, the results are presented based on the dimensionless time $\left(t^{*}=\frac{t U_{0}}{D_{d}}\right)[13]$.

Table 1 - The simulation parameters for the different cases (SI units are used).

\begin{tabular}{c|cccccccccccc}
\hline case & C-1 & C-2 & C-3 & C-4 & C-5 & C-6 & C-7 & C-8 & C-9 & C-10 & C-11 & C-12 \\
\hline $\mathrm{U}_{0}$ & 0.1 & 0.25 & 0.5 & 1 & 0.1 & 0.25 & 0.5 & 1 & 0.1 & 0.25 & 0.5 & 1 \\
$R$ & 1 & 1 & 1 & 1 & 1.5 & 1.5 & 1.5 & 1.5 & 0.66 & 0.66 & 0.66 & 0.66 \\
We & 11.1 & 69.4 & 277.7 & 1111.1 & 11.1 & 69.4 & 277.7 & 1111.1 & 7.7 & 48.6 & 194.4 & 777.7
\end{tabular}
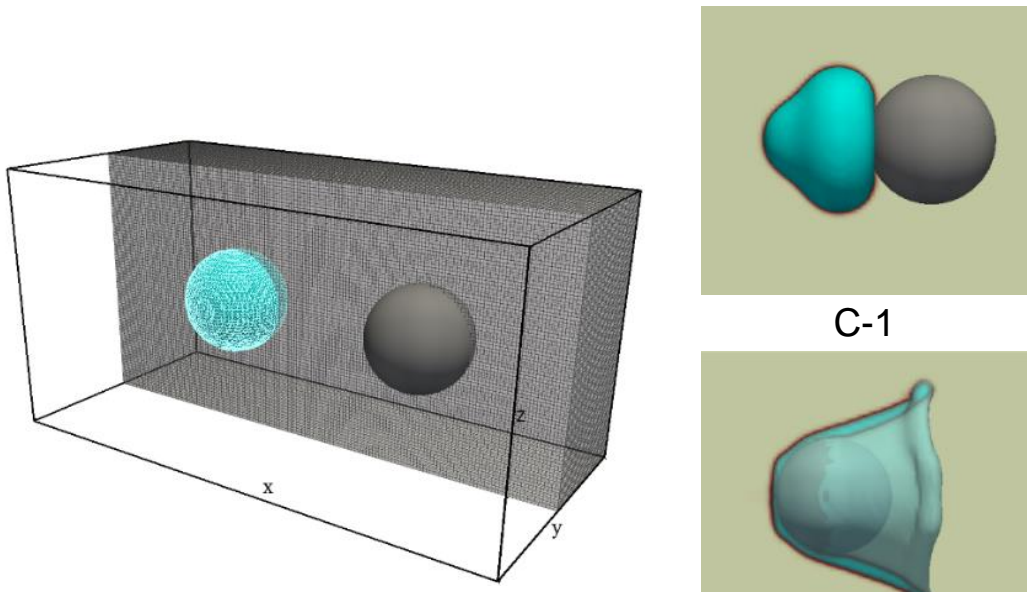

C-1

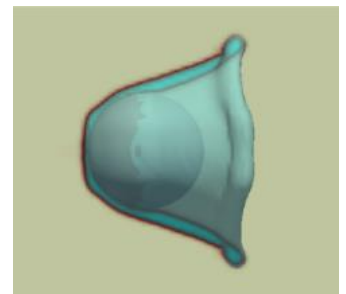

C-3

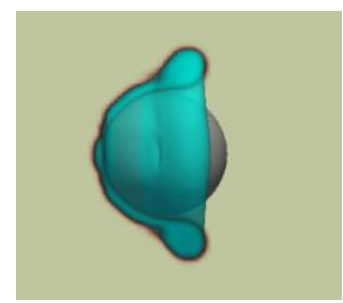

C-2

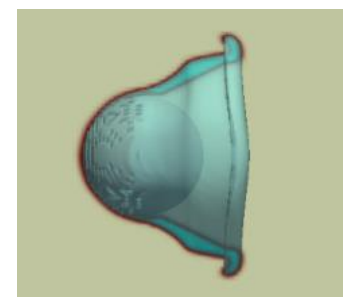

C-4

Figure 3. Simulation setup for the droplet-particle collision (left), and the snapshots of droplet-particle interaction at $t^{\star}=1$ after the collision for $\mathrm{R}=1$ with different We numbers (right).

\section{Results and Discussion}

First, we analyse the physics of collision outcome based on the instantaneous flow fields. Figure 3 shows the snapshots of the droplet-particle interaction at $t^{*}=1$ after the collision for cases $\mathrm{C}-1$ to $\mathrm{C}-4(\mathrm{R}=1)$. The outcome is clearly controlled by the competition between surface tension force and kinetic energy upon impact. For C-1 with the lowest We, the droplet kinetic energy could not overcome the surface tension's consolidating effect, thus, the droplet does not spread over the particle and rebounds. This behaviour is known for the low We number collisions [13][17]. As We number increases, the droplet starts spreading on the particle and 
undergoes severe deformations. A lamella with a rim is formed with the height and diameter determined by the outcome of the force balance. For C-2, the lamella height does not exceed the particle equator and results in a thick rim, whereas at higher We (C-3 and C-4) a longer but thinner lamella is formed whose rim is prone to disintegrate further downstream. Such a difference in the rim shape is also noticed in [15] for the We $>100$. In fact, the level of kinetic energy is excessively higher than the surface tension and dissipative forces against droplet spreading, and as we later show, the thin film over the particle will eventually rupture. It has to be noted that this interfacial physics might have remained under-resolved in this simulation as the thickness of such a thin film is controlled by the grid resolution.
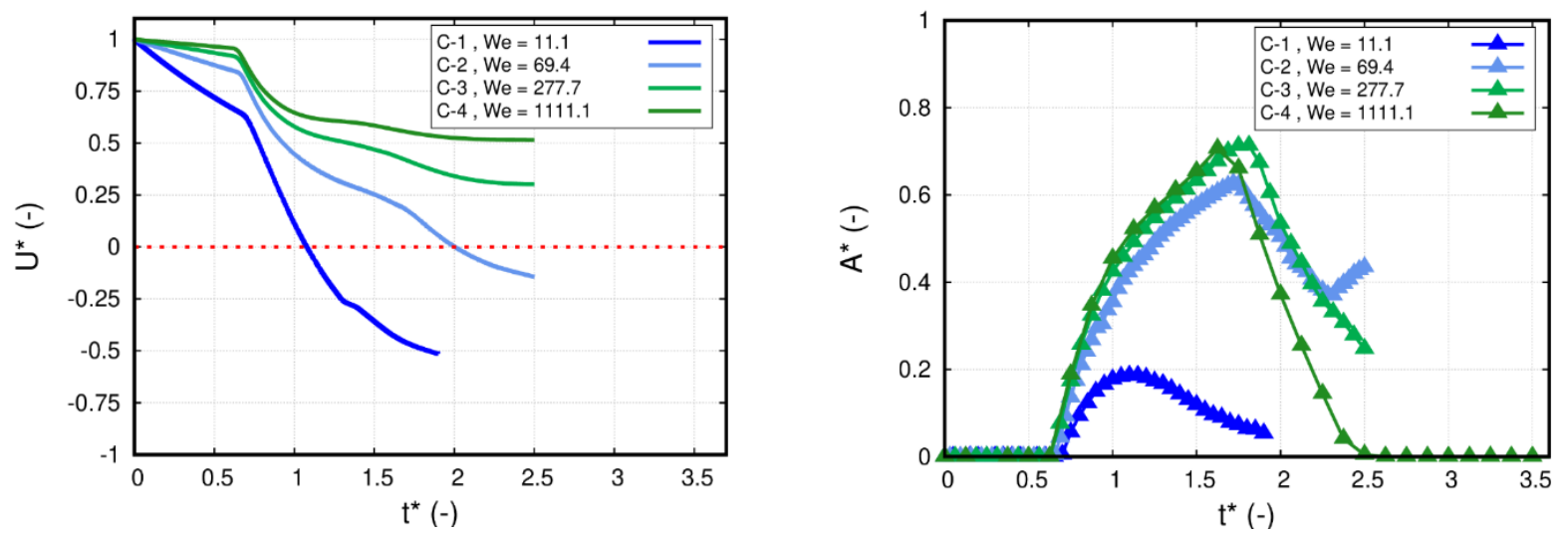

Figure 4. The particle dimensionless velocity (left) and wetted area (right) for $R=1$ at different We numbers.
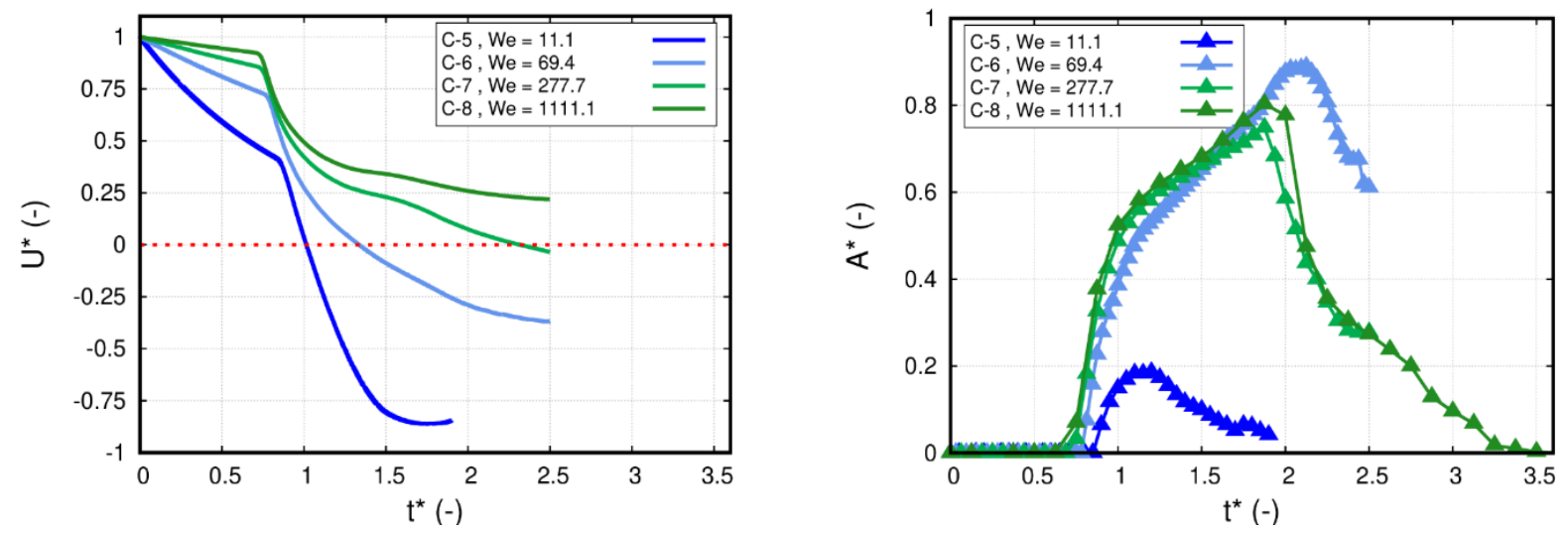

Figure 5. The particle dimensionless velocity (left) and wetted area (right) for $R=1.5$ at different We numbers.
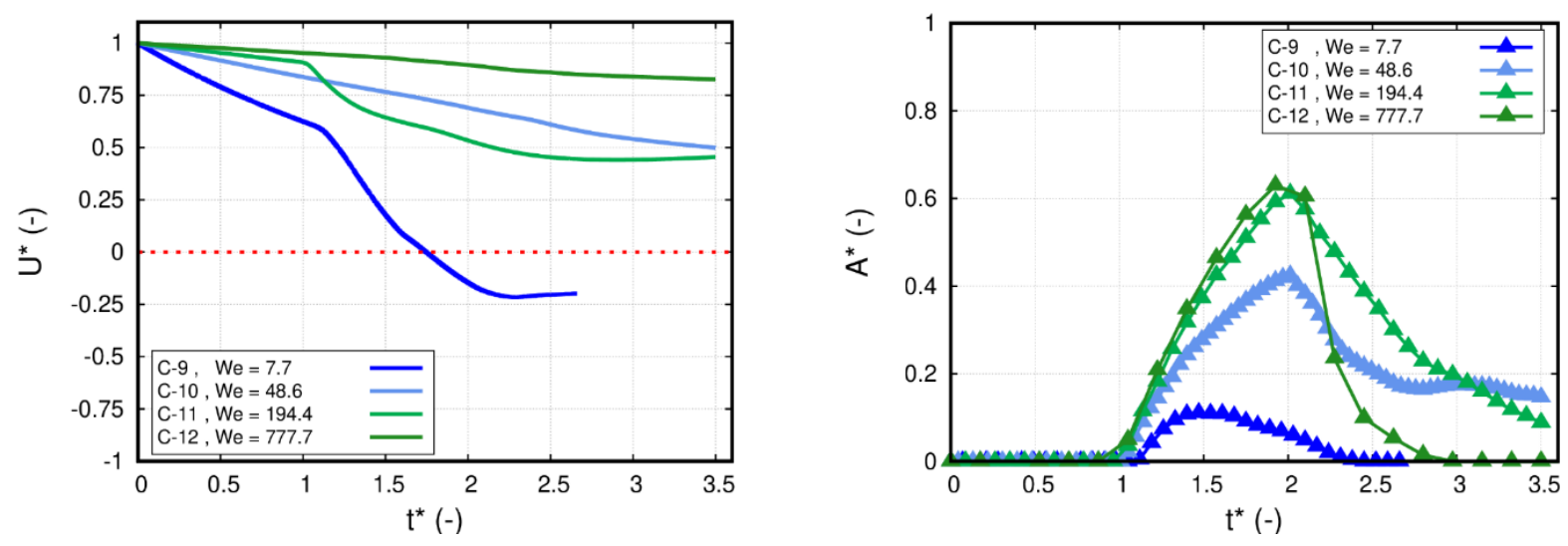

Figure 6. The particle dimensionless velocity (left) and wetted area (right) for $R=0.66$ at different We numbers. 
According to [13], there are two major regimes for We numbers up to 80 , namely, rebound and coating. Similar to their approach, we quantify (I) the velocity of the particle centroid along the collision axis $\left(U_{p, x}\right)$, and (II) the integrated area covered by liquid $(A)$. Then, the dimensionless particle velocity $U^{*}=U_{p, x} / U_{0}$ and dimensionless wetted area $A^{*}=A / A_{p}$ are used for analysis. Figures 4 to 6 display the temporal evolution of $U^{*}$ and $A^{*}$ for various We numbers at each $R$. The $U^{*}=0$ line is plotted to determine possible rebound mode. For all the cases, the collision happens at $t^{*} \approx 0.7$. For the cases with $\mathrm{R}=1$ (Figure 4 ), the rebound regime is evident at the lowest We number (C-1) as the particle velocity can reach up to $50 \%$ of its initial velocity in opposite direction. In C-2, the droplet spreads on the particle while reducing particle velocity and keeps almost half of the particle area wet for $t^{*}>1$. This corresponds to the coating regime as also reported by [13] for $40<\mathrm{We}<80$. At We $>250$, the droplet first wets the particle and forms a lamella as also shown in Figure 3. However, the lamella disintegrates at almost 1 dimensionless time after the collision $\left(t^{*} \approx 1.7\right)$. Therefore, the particle continues its free motion with a constant velocity as evident in Figure 4. The lamella fragmentation also causes a sudden decrease in $A^{*}$ for both C-3 and C-4. At the largest We number, the kinetic energy level is so high that until $t^{*}=2.5$ all the droplet volume is washed away from the particle. Even though in C-3 there is still some liquid hold-up, it eventually should turn to zero as the particle keeps moving with constant velocity. So for the cases with $\mathrm{We}>250$, the partial-coating and complete wash-off modes can be characterized by the simulations. The results for $R>1$ reveal a similar trend but with a stronger tendency towards coating as reflected in the larger peaks for $A^{*}$. Because with higher droplet volume, a thicker lamella is formed which is resistant enough against breakup. Therefore, for $\mathrm{C}-6$ almost $60 \%$ of the particle area remains coated by the droplet, and at higher We numbers (C-7 and C8), a delay occurs for the wash-off mode as demonstrated in Figure 5. The results also reveal the tendency against coating for $R<1$ as the maximum of $A^{*}$ for all the We numbers reduces significantly (Figure 6 ), and even with a moderate We number ( $\mathrm{C}-10)$ the coating regime is not observed due to the smaller droplet volume. Figure 6 also shows that except for the rebound regime of C-9, the particle velocity in other cases is not influenced by the collision as the droplet is smaller and could be pushed more easily.

\section{Conclusions}

We present a VOF-based fictitious domain method (resolved CFD-DEM) for the fully-resolved simulation of the liquid-gas interfacial flow interacting with solid particles. While the fluid-fluid interface is captured by the VOF in an Eulerian domain, an explicit penalty term is added to the momentum equation to account for particle presence. First, the method is validated with an experimental benchmark problem. Then, the droplet-particle binary collision is simulated at different conditions characterized by Weber number and droplet-to-particle diameter ratio. Besides the rebound and coating regimes reported by previous works, we could observe some new modes such as partial coating and sudden wash-off at a higher range of Weber numbers $(250<W e<1100)$. Nevertheless, there remains some under-resolved interfacial physics as well as some modelling shortcomings for future work. This approach does not consider particle wettability. Even though the high-speed collision in most of the simulations in this study may outweigh the effect of surface wetting, accurate prediction of the wetting phenomenon is essential for moderate We number cases where the physics of thin film and the time-scales required for coating becomes important. The implementation of a model for contact line dynamics at the particle surface is the subject of our ongoing research. Future work will focus on the development of multiscale methods based on the resolved and unresolved VOF-DEM. 


\section{Nomenclature}

$\alpha \quad$ volume of fluid [-]

$\Phi$ particle volume fraction [-]

D diameter [m]

$\rho \quad$ density $\left[\mathrm{kg} \mathrm{m}^{-3}\right]$

$\mu \quad$ dynamic viscosity $\left[\mathrm{kg} \mathrm{m}^{-1} \mathrm{~s}^{-1}\right]$

$\sigma \quad$ surface tension $\left[\mathrm{kg} \mathrm{s}^{-2}\right]$

$\mathrm{U} \quad$ velocity $\left[\mathrm{m} \mathrm{s}^{-1}\right]$

$\mathrm{K} \quad$ permeability of solid region [-]

We Weber number [-]

$\mathrm{R} \quad$ droplet-to-particle diameter ratio [-]

\section{References}

[1] Charalampous, G., Hardalupas, Y., 2017, Physics of Fluids, 29, pp. 103305.

[2] Rafidi, N., Brogaard, F., Chen, L., Hakansson, R., Tabikh, A., 2018, sustainable environment research, 28, pp. 382-388.

[3] Kieckhefen, P., Lichtenegger, T., Pietsch, S., Pirker, S., Heinrich, S., 2019, Particuology, 42, pp. 92-103.

[4] Khojasteh, D., Moradi Kazerooni, N., Marengo, M., 2019, Journal of Industrial and Engineering Chemistry, 71, pp. 50-64.

[5] Peskin, C., 1972, Journal of Computational Physics, 10, pp. 252-271.

[6] Mittal, R., laccarino, G., 2005, Annual Review of Fluid Mechanics, 37, pp. 239-261.

[7] Engels, T., Kolomenskiy, D., Schneider, K., Sesterhenn, J., 2015, Journal of Computational Physics, 10, pp. 96-115.

[8] Deen, N.G., van Sint Annaland, M., Kuipers, J., 2009, Chemical Engineering Science, 64, pp. 2186-2201.

[9]Yoon, H.S., Jeon, C.H., Jung, J.H., Koo, B., Choi, C., Shin, S.H., 2013, International Journal for Numerical Methods in Fluids, 73, pp. 250-265.

[10] Sun, X., Sakai, M., 2016, Chemical Engineering Science, 139, pp. 221-240.

[11] Patel, H.V., Das, S., Kuipers, J., Padding, J.T., Peters, E.A.J.F., 2017, Chemical Engineering Science, 166, pp. 28-41.

[12] O'Brien, A., Bussmann, M., 2020, Journal of Computational Physics, 402, pp. 109089.

[13] Malgarinos, I., Nikolopoulos, N., Gavaises, M., 2016, International Journal of Heat and Fluid Flow, 61, pp. 499-509.

[14] Bordbar, A., Taassob, A., Khojasteh, D., Marengo, M., Kamali, R., 2018, Langmuir, 34, pp. 5149-5158.

[15] Vilela, V., de Souza, F.J., 2020, Flow, Turbulence and Combustion, 105, pp. 965-987.

[16] Milacic, E., Baltussen, M.W., Kuipers, J., 2019, Powder Technology, 354, pp. 11-18.

[17] Yoon, I., Shin, S., 2021, International Journal of Multiphase Flow, 135, pp. 103503.

[18] Pozzetti, G., Peters, B., 2018, International Journal of Multiphase Flow, 99, pp. 186-204.

[19] Saeedipour, M., 2019, Sep 2-4, 2019, 29th Conference on Liquid Atomization and Spray Systems.

[20] Kloss, C., Goniva, C., Hager, A., Amberger, S., Pirker, S., 2012, Progress in

Computational Fluid Dynamics, 12, pp. 140-152.

[21] Hager, A., Kloss, C., Pirker, S., Goniva, C., 2014, Journal of Computational Multiphase Flows, 6, pp. 13-27.

[22] Specklin, M., Delaure, Y., 2018, European Journal of Mechanics-B/Fluids, 70, pp. 130147.

[23] Aristoff, M.J., Truscott, T.T., Techet, A.H., Bush, J.W.M., 2010, Physics of Fluid, 22, pp. 032102.

[24] Mirzaii, I., Passandideh-Fard, M., 2012, International Journal of Multiphase Flow, 39, pp. 216-226. 